\title{
A Simple Classification System for Hip Fractures
}

\author{
Kareem Elsorafy1 ${ }^{*}$, Ahmad Machaurab1', Tamer Kamal'2, Sherief El-Nikety³, Sunny Deo1 \\ ${ }^{1}$ Department of Trauma and Orthopaedics at the Great Western Hospital, Swindon, UK \\ ${ }^{2}$ Department of Trauma and Orthopaedics at the Robert Jones and Agnes Hunt Orthopaedic Hospital, \\ Oswestry, UK \\ ${ }^{3}$ Department of Trauma and Orthopaedics at the Royal National Orthopaedics Hospital, London, UK \\ Email: *kelsorafy@gmail.com
}

Received 11 April 2014; revised 16 May 2014; accepted 23 May 2014

Copyright (C) 2014 by authors and Scientific Research Publishing Inc.

This work is licensed under the Creative Commons Attribution International License (CC BY). http://creativecommons.org/licenses/by/4.0/

(c) (i) Open Access

\section{Abstract}

Background: We have developed a Hip Fracture Classification which stratifies patients into 4 groups upon admission according to fracture complexity. This classification considers pathoanatomic and physiologic parameters and was developed to help identify high-risk patients, with more surgical demands and consequently higher incidence of morbidity. Materials and Methods: Data was gathered prospectively for a cohort of 273 consecutive patients admitted over a 12month period between 2008 and 2009 and classified according to the Hip Fracture Complexity Classification (CO - C3). The following outcome measures (mortality and length of hospital stay) were reviewed at thirty days and one year. Result: The overall mortality was $4.4 \%$ at 30 days and $20 \%$ at 1 year. There were significant differences in 1-year mortality between all stratified groups $(P<0.001)$, but not at 30 -days. No difference in length of hospital stay was noted between complexity groups $(P=0.4)$. Conclusion: Clinical complexity is a significant factor influencing patient outcomes following hip fractures. Our classification helps highlight patients with higher surgical demands, aiming to draw more resources and help with optimization of care to those candidates. We believe such a system could be used to evaluate different hospitals performance more accurately. Since, national data is published annually; this system can help correlate morbidity and mortality with clinical complexity.

\section{Keywords}

Hip Fracture, Mortality, Death, Classification, Stratification

\footnotetext{
${ }^{*}$ Corresponding author.
} 


\section{Introduction}

With an increase in the incidence of hip fractures due to population aging, there were nearly 76,000 cases of hip fractures between 2009-2010, amounting to 1.4 billion British pounds of total expenditure a year [1]. Historically, a proportion of patients with hip fractures received poor quality of care; patients usually suffer from a number of coexisting comorbidities; and the injury is associated with a high incidence of morbidity and mortality. Mortality figures range from $5 \%-10 \%$ at 30 days postoperatively and $19 \%-33 \%$ at 1 year [2] [3].

The National Hip Fracture Database (NHFD) provides polices and guidelines for all UK hospitals within the National Health Service (NHS). This database started in September 2007 in collaboration with the British Orthogeriatric Society and the British Orthopaedic Association. The NHS financing is based on payment by results. Every hospital is responsible for gathering data about its annual performance, which is published yearly by the NHFD in a transparent manner. Since the inception of the best practice tariff, there has been an on-going reduction in national mortality overall. The current system may not differentiate between more complicated hip fracture cases associated with medical and surgical complexity, and consequently they are at a higher risk of having poorer outcomes and others are with less complex cases for which a better outcome should be expected.

Much research has focused on individual causes of poor results from surgery following hip fractures. Very few sought to assess the effects of cumulative complexity and compare them to noncomplex groups. We observed that patients with multiple significant comorbidities and significant fracture patients are at highest risk of complications and fatality. Therefore at our institution, we developed a simple classification system to help stratify hip fracture patients according to their complexity to aid identification of high-risk patients and prioritisation of medical care and resources while planning their anaesthetic needs and surgical expertise required.

\section{Materials and Methods}

This is a prospective observational study of a consecutive cohort of hip fracture patients admitted to our institution, which is a large district general hospital. Patients aged 60 years old and above were included in our study in the twelve-month period from June 2008 to June 2009. Data was gathered prospectively at our weekly multidisciplinary audit meeting attended by orthogeriatricians, orthopaedic surgeons, nurses, therapist and theatre staff using a database system. The prospective data collected included:

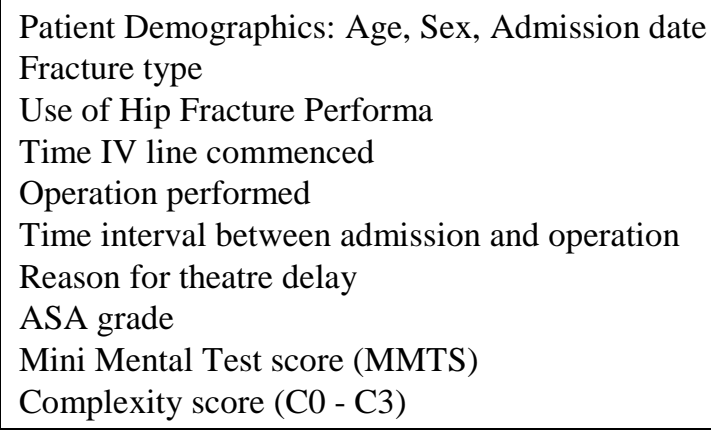

In order to calculate the complexity classification, either on admission or at the following morning trauma meeting, 2 questions were asked.

1) Is there local complexity at the fracture site?

2) Is the patient systemically complex?

The combination of yes/no for each response provides the 4 potential options of the classification.

- Co are medically fit and well patients with simple straightforward fractures. They are ASA (American Society of Anesthesiologists) 1 or 2 i.e., straightforward. (NO/NO)

- C1 are fit patients but with complex fractures such as those with multi-fragmentary unstable fracture patterns or pathologic fractures. In addition any other bone or soft tissue abnormality which increases the surgical time or risk of local complication is also included, such as bony dysplasia, previous radiotherapy to area i.e., locally complex. (Yes/NO)

- $\quad \mathbf{C 2}$ are Unwell patients with a simple fracture i.e., systemically complex. (No/Yes)

- C3 are Unwell and with a Complex fracture i.e., locally and systemically complex. (Yes/Yes) 
limOur working definition of "unfit" is having one or more un-controlled co-morbidities, three or more controlled co-morbidities as defined for guidelines for venous thromboembolism [4] or an ASA score of $\geq 3$ or a Mini Mental Test score (MMTS) of $\leq 7$ [4]. The definition of generally fit and well is therefore patients with no co-morbidities or one or two controlled co-morbidities, ASA $\leq 2$ and a MMTS of $\geq 8$. While Complex fractures are either $\geq 3$ part fractures or subtrochanteric extension or pathologic fractures.

Using our electronic patients' record system length of hospital stay and mortality were identified.

Our key outcome measures were mortality at 30 days and 1 year and length of hospital stay.

The research and development department classified our work as service evaluation, hence no ethical committee approval was required and audit approval was granted from our institution audit department.

\section{Statistical Analysis}

All data was entered into Microsoft Excel spread sheets. The principle statistical tools used were Excel pivot tables and InStat 3. A $P$ value of $<0.05$ was considered statistically significant and the confidence interval was set to $95 \%$.

\section{Results}

Two hundred and ninety patients were admitted during our study period, patients below the age of 60 and patients with incomplete data entries were excluded. 273 patients were included in this study. Testing data using the stem and leaf plot showed that the data is parametric. The mean age of our study group was 81.3 years (range of 60 - 102 with a standard deviation of 8.63). Eighty percent were females. A summary of the number of patients in each complexity group is summarised in a pie chart 1 and basic demographic data is summarised in Figure 1. Basic demographic differences between complexity groups are summarised in Table 1.

The 30 -day mortality was $4.4 \%$ compared to a national average of $7.7 \%$ published by the NHFD during the same period. The one-year mortality was $20 \%$ with no national average to compare to at the same period. However, a recent paper published from Nottingham had an annual mortality average of $29.3 \%$ [2].

The 30-day mortality was stratified by complexity groups, analysed using the Chi-square for trend, failed to show statistical significance ( $P<0.05$ at a $95 \%$ confidence interval) and the one-year mortality stratified by complexity groups was statistically significant $(P<0.001$ at a $95 \%$ confidence interval) (Figure $2 \&$ Figure 3).

Table 1. Basic demographic difference and outcomes stratified by Swindon Complexity.

\begin{tabular}{ccccc}
\hline & C0 & C1 & C2 & C3 \\
\hline Number & $121(44.3 \%)$ & $21(7.7 \%)$ & $93(34.1 \%)$ & $38(13.9 \%)$ \\
Average Age & 79.7 & 72.23 & 80 & 83.4 \\
F $:$ M & $100: 21$ & $13: 8$ & $64: 29$ & $36: 2$ \\
30 day mortality & 1 & 2 & 3 & 4 \\
1 year mortality\% & $8(6.6 \%)$ & $2(9.5 \%)$ & $23(24.7 \%)$ & $15(39.5 \%)$ \\
Length of hospital stay & 18.5 & 19 & 26 & 31 \\
\hline
\end{tabular}

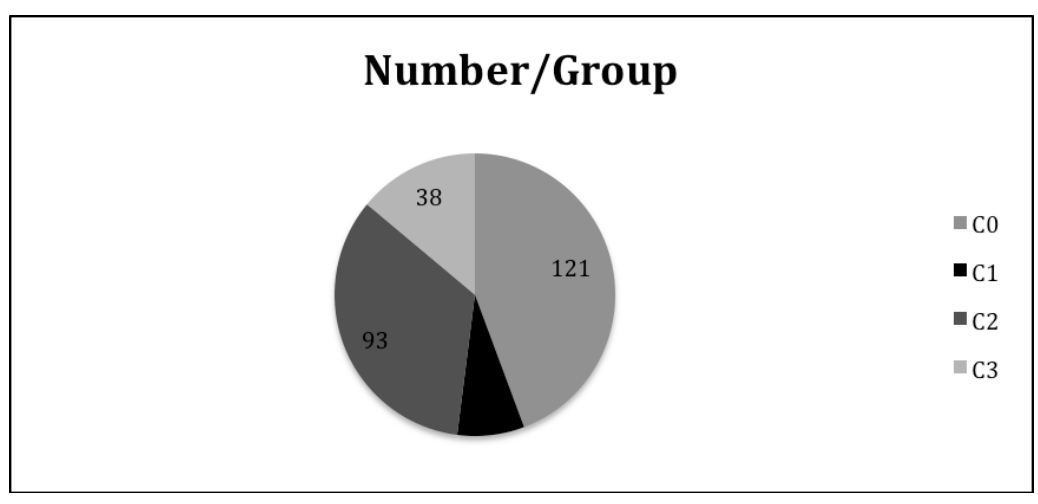

Figure 1. The number of patients within each of the complexity groups. 


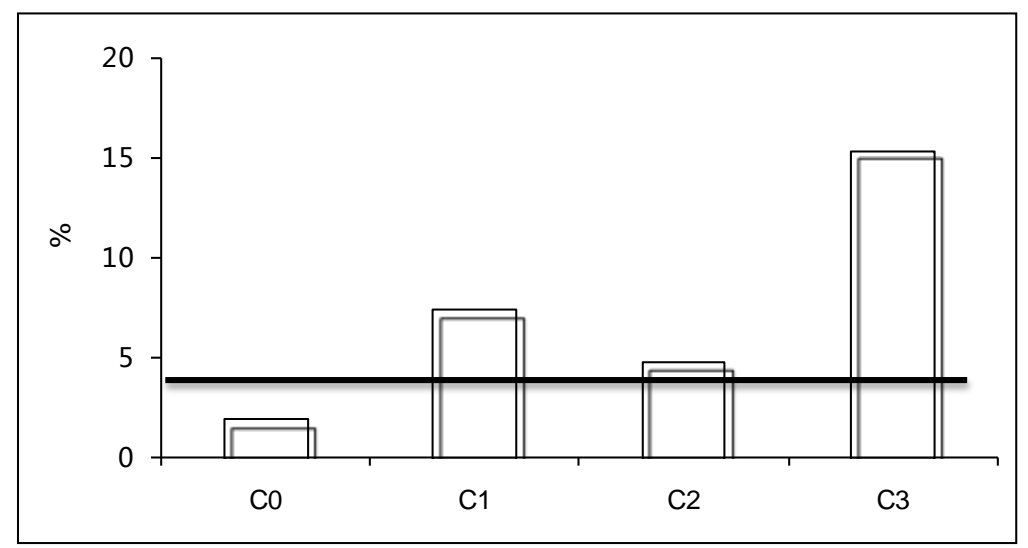

Figure 2. 30-day mortality rate, line represents mean.

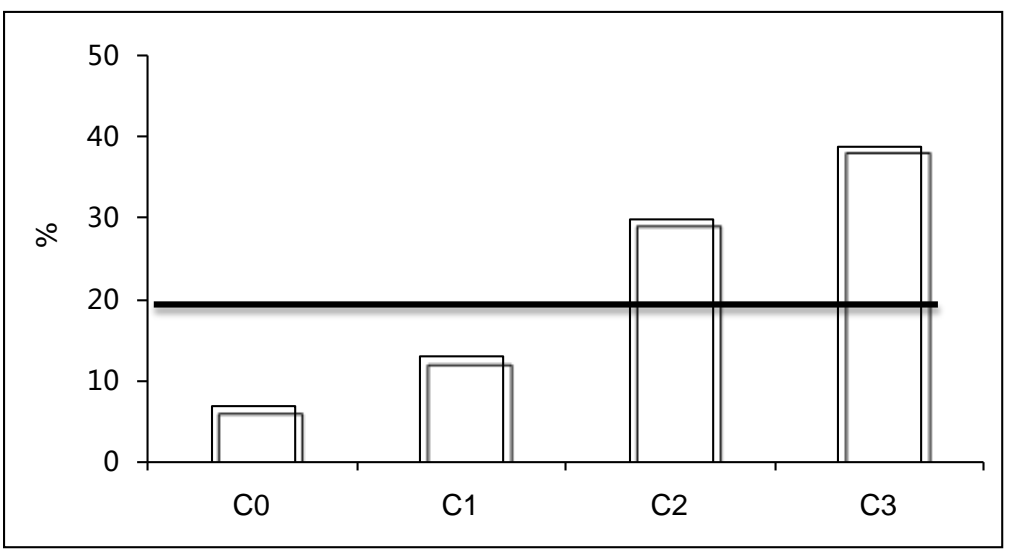

Figure 3. 1-year mortality rate, line represents the mean.

There was a higher risk in patients with more complex fracture patterns.

Mortality stratified by complexity did show a linear trend, with an increasing mortality in more complex groups.

The mean length of stay was 23 days (range 1 - 124 days, sd 20.17 and std. error of 1.22). When length of hospital stay was stratified by complexity and analysed for non-parametric data no statistically significant difference was found ( $P$ 0.39), but a linear trend was noted as shown in Figure 4.

\section{Discussion}

Our classification considers pathoanatomic injury factors and overall patient status. It is bases on what we encounter in clinical practice and on clinical observation. Maxwell et al., developed the Nottingham Hip Fracture Score as a predictor of the risk mortality by considering the following physiologic parameters; Age, Sex, Admission Haemoglobin, Admission MMTS, Number of co-morbidities, presence of malignancy, and if patients lived in an institution [5]. After a score is worked, a risk of mortality is calculated against a graph. These parameters lack the stratification of the anatomical injury, which may translate to a more severe physiologic insult, as occurs in higher energy injuries. Surgical insult is significant to frail neck of femur patients and the more complex a fracture, the longer the operation, and therefore adds further insult to the patients' physiologic systems.

POSSUM score (Physiological and Operative Severity Score) developed by Copeland et al. and modified for NOF fractures by Ramanathan et al. is a complex scoring system that looks at 12 physiologic parameters and surgical parameters, the ones applicable to hip fractures are: type and number of operative procedures, amount of blood loss, presence of malignancy [6] [7]. In the latter scores, the surgical score is almost the same for every patient with a NOF fracture, reducing the value of this scoring system. 


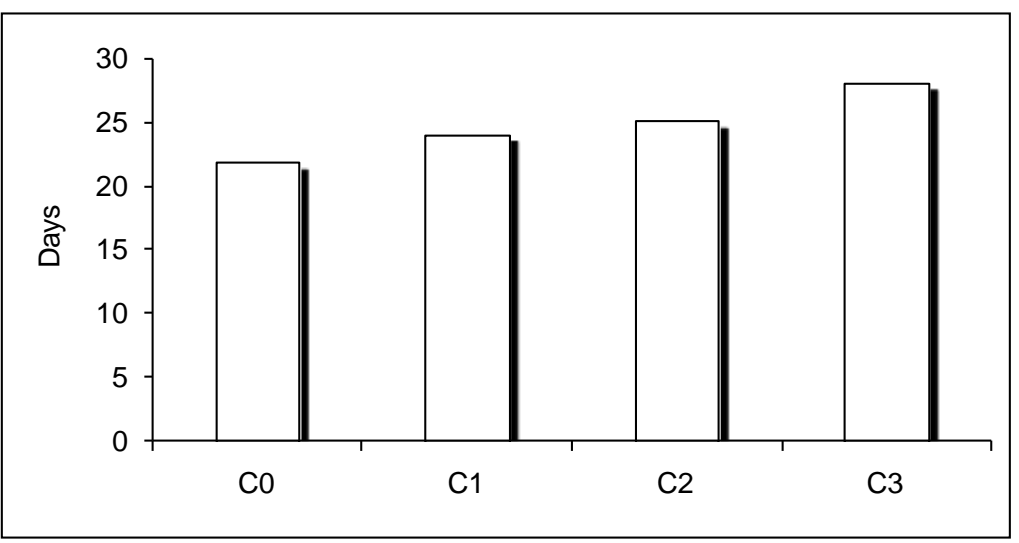

Figure 4. Length of hospital stay stratified by complexity.

The relationship between low MMTS and higher mortality figures has been supported by a number of publications [8] [9]. Beloosesky et al. and Huusko et al. opposed the correlation between low MMTS and increased mortality [5] [10]-[12]. As our unit mortality in the first 30-days was 10 patients, further subgroup analysis to this small number was deemed of no statistical value.

There is some controversy between the value of the ASA grade and predicting mortality in the literature [5] [13] [14]. Some authors support the link between ASA score and mortality [15]-[20] (Dzupa 2002; Bjorgul 2010; Batsis 2007; Al-Arabi2009; Talsnes 2011; Söderqvist 2009) and our findings have shown a significant correlation between ASA group and mortality. To minimise controversy about the ASA, we have not solely relied on ASA to indicate systemic complexity and therefore, considered the number of co-morbidities and the presence of a single chronic uncontrolled comorbidity to reflect systemic complexity.

The Donati score is a non-specific general surgical and orthopaedic 4-item score using age range, ASA, surgical severity, and urgency of surgery to calculate mortality risk. Again, all NOF fractures are deemed urgent.

We feel that our classification system is useful clinically as it summarises information gathered in the Neck of Femur admission pathway rapidly and can be simply applied by Orthopaedic or Orthogeriatric doctors upon admission in real time. The classification can then be used in communication with all members of the multi-disciplinary team along a patients care pathway. Our study demonstrates increasing mortality risk and therefore confines its utilisation in identifying risk and prioritisation of medical and surgical management both in terms of timing and seniority to help achieve the best outcome.

This classification allows a simple easy method of communication between surgeons, anaesthetists, orthogeriatricians and nurses allowing high-risk patients to be identified early and the start of immediate optimisation of care. Expanding the use of this system may provide prognostic indicators.

An additional potential use of the classification is that it could help differentiate between a hospital performing well with a fitter cohort of hip fracture patients (i.e., more $\mathrm{C} 0$ ) and other hospitals that may have more complex patients. We previously used a similar complexity classification system for our elective knee replacement patients [15].

We hope to address some weakness of this study in the future namely looking at complications and readmissions in relation to increasing complexity, using our database to score the other available scoring systems to compare with other complexity scores and to increase the numbers of patients in the cohort group over time.

\section{Conclusion}

This hip fracture complexity classification is simple to use and implement. It reflects the clinical complexity of hip fracture patients, which allows identification of clinical risk and therefore appropriates allocation of resources. It could potentially allow a more accurate comparison of mortality between different hospitals if used nationally.

\section{Key Points}

- Complexity Classification correlates with Hip fractures Mortality. 
- Complexity Classification helps stratify patient care.

- Complexity Classification can provide better information on hospital performance.

\section{Acknowledgments}

We acknowledge that we have not received any grants or source of funding to produce any parts of the above project.

\section{References}

[1] Currie, C., Fleming, S. and Partridge, M. (2010) The National Hip Fracture Database National Report, 2010. The National Hip Fracture Database.

[2] Wiles, M.D., Moran, C.G., Sahota, O. and Moppett, I.K. (2011) Nottingham Hip Fracture Score as a Predictor of One Year Mortality in Patients Undergoing Surgical Repair of Fractured Neck of Femur. British Journal of Anaesthesia, 106, 501-504. http://dx.doi.org/10.1093/bja/aeq405

[3] Roche, J.J.W., Wenn, R.T., Sahota, O. and Moran, C.G. (2005) Effect of Comorbidities and Postoperative Complications on Mortality after Hip Fracture in Elderly People: Prospective Observational Cohort Study. BMJ: British Medical Journal, 331, 1374. http://dx.doi.org/10.1136/bmj.38643.663843.55

[4] Duggan-Keen, M. (2010) Reducing the Risk of VTE: The New NICE Clinical Guideline. Nurse Prescribing, 8, 589-594.

[5] Maxwell, M.J., Moran, C.G. and Moppett, I.K. (2008) Development and Validation of a Preoperative Scoring System to Predict 30 Day Mortality in Patients Undergoing Hip Fracture Surgery. British Journal of Anaesthesia, 101, 511-517. http://dx.doi.org/10.1093/bja/aen236

[6] Copeland, G.P., Jones, D. and Walters, M. (2005) POSSUM: A Scoring System for Surgical Audit. British Journal of Surgery, 78, 355-360. http://dx.doi.org/10.1002/bjs.1800780327

[7] Ramanathan, T.S., Moppett, I.K., Wenn, R. and Moran, C.G. (2005) POSSUM Scoring for Patients with Fractured Neck of Femur. British Journal of Anaesthesia, 94, 430-433. http://dx.doi.org/10.1093/bja/aei064

[8] Van Dortmont, L.M.C., Douw, C.M., van Breukelen, A.M.A., et al. (2000) Outcome after Hemi-Arthroplasty for Displaced Intracapsular Femoral Neck Fracture Related to Mental State. Injury, 31, 327-331. http://dx.doi.org/10.1016/S0020-1383(99)00304-6

[9] Panula, J., Pihlajamäki, H., Mattila, V.M., et al. (2011) Mortality and Cause of Death in Hip Fracture Patients Aged 65 or Older-A Population-Based Study. BMC Musculoskeletal Disorders, 12, 105. http://dx.doi.org/10.1186/1471-2474-12-105

[10] Huusko, T.M., Karppi, P., Avikainen, V., Kautiainen, H. and Sulkava, R. (2000) Randomised, Clinically Controlled Trial of Intensive Geriatric Rehabilitation in Patients with Hip Fracture: Subgroup Analysis of Patients with Dementia. BMJ: British Medical Journal, 321, 1107. http://dx.doi.org/10.1136/bmj.321.7269.1107

[11] Ooi, L.H., Wong, T.H., Toh, C.L. and Wong, H.P. (2005) Hip Fractures in Nonagenarians-A Study on Operative and Non-Operative Management. Injury, 36,142-147. http://dx.doi.org/10.1016/j.injury.2004.05.030

[12] Beloosesky, Y., Grinblat, J., Epelboym, B. and Hendel, D. (2001) Dementia Does Not Significantly Affect Complications and Functional Gain in Elderly Patients Operated on for Intracapsular Hip Fracture. Archives of Orthopaedic and Trauma Surgery, 121, 257-260. http://dx.doi.org/10.1007/s004020000220

[13] Owens, W.D., Felts, J.A., and Spitznagel Jr., E.L. (1978) ASA Physical Status Classifications: A Study of Consistency of Ratings. Anesthesiology, 49, 239. http://dx.doi.org/10.1097/00000542-197810000-00003

[14] Burgos, E., Gómez-Arnau, J.I., Diez, R., Munoz, L., Fernández-Guisasola, J. and Garcia del Valle, S. (2008) Predictive Value of Six Risk Scores for Outcome after Surgical Repair of Hip Fracture in Elderly Patients. Acta Anaesthesiologica Scandinavica, 52, 125-131. http://dx.doi.org/10.1111/j.1399-6576.2007.01473.x

[15] Al-Arabi, Y.B. (2009) Risk Classification for Primary Knee Arthroplasty. The Journal of Arthroplasty, 24, 90-95. http://dx.doi.org/10.1016/j.arth.2008.02.010

[16] Dzupa, V., Bartonícek, J., Skála-Rosenbaum, J. and Príkazský, V. (2002) Mortality in Patients with Proximal Femoral Fractures during the First Year after the Injury. Acta chirurgiae orthopaedicae et traumatologiae Cechoslovaca, 69, 39-44.

[17] Talsnes, O., Hjelmstedt, F., Dahl, O.E., Pripp, A.H. and Reikerås, O. (2011) Clinical and Biochemical Prediction of Early Fatal Outcome Following Hip Fracture in the Elderly. International Orthopaedics, 35, 903-907. http://dx.doi.org/10.1007/s00264-010-1149-7

[18] Bjorgul, K., Novicoff, W.M. and Saleh, K.J. (2010) American Society of Anesthesiologist Physical Status Score May 
Be Used as a Comorbidity Index in Hip Fracture Surgery. The Journal of Arthroplasty, 25, 134-137.

[19] Söderqvist, A., Ekström, W., Ponzer, S., Pettersson, H., Cederholm, T., Dalén, N., et al. (2009) Prediction of Mortality in Elderly Patients with Hip Fractures: A Two-Year Prospective Study of 1,944 Patients. Gerontology, 55, 496-504.

[20] Batsis, J.A., Phy, M.P., Melton, L.J., Schleck, C.D., Larson, D.R., Huddleston, P.M., et al. (2007) Effects of a Hospitalist Care Model on Mortality of Elderly Patients with Hip Fractures. Journal of Hospital Medicine, 2, 219-225. http://dx.doi.org/10.1002/jhm.207 